\title{
Gasdynamic wave interaction in two spatial dimensions
}

\author{
By S. R. SANDERSON† \\ Graduate Aeronautical Laboratories, California Institute of Technology, Pasadena, CA 91125, USA
}

(Received 6 March 2003 and in revised form 20 November 2003)

We examine the interaction of shock waves by studying solutions of the twodimensional Euler equations about a point. The problem is reduced to linear form by considering local solutions that are constant along each ray and thereby exhibit no length scale at the intersection point. Closed-form solutions are obtained in a unified manner for standard gasdynamics problems including oblique shock waves, PrandtlMeyer flow and Mach reflection. These canonical gas dynamical problems are shown to reduce to a series of geometrical transformations involving anisotropic coordinate stretching and rotation operations. An entropy condition and a requirement for geometric regularity of the intersection of the incident waves are used to eliminate spurious solutions. Consideration of the downstream boundary conditions leads to a formal determination of the allowable downstream matching criteria. By retaining the time-dependent terms, an approach is suggested for future investigation of the open problem of the stability of shock wave interactions.

\section{Introduction}

Riemann problems in two spatial dimensions are germane to many problems in experimental, analytical and computational gasdynamics. Familiar examples include the interaction of oblique shock waves in steady supersonic flow, the self-similar problem of transition to Mach reflection from a wedge and the treatment of discontinuous shock fronts in numerical shock fitting algorithms.

The well-known approach to the interaction of shock waves has its basis in the work of Rankine (1870) and Hugoniot (1889) regarding the propagation of shock waves. The solution for oblique shock waves was then obtained by Meyer (1908) through a Galilean transformation that imposed a velocity component parallel to the shock front. Also due to Meyer is the development of the Prandtl-Meyer function for expansion through an expansion fan. The development of the theory of shock wave interactions by Courant, Friedrichs, von Neumann and independently by Weise is described by Courant \& Friedrichs (1948). By hypothesizing the existence of a vortex sheet at the intersection point, solutions for several interacting shock waves and/or expansion fans may be constructed that are consistent with the experimental observations. A similar approach has been developed for shock-shear layer interactions. The interaction solutions are represented graphically by the intersection in the pressure-flow deflection angle, $p-\delta$, plane of the loci of possible downstream states for the interacting waves. Because of the nonlinear interaction of the waves, problems of non-uniqueness

$\dagger$ Present address: GE Global Research, 1 Research Circle, Niskayuna, NY 12309, USA. 
and non-existence of solutions arise, with the Mach reflection problem being one classic example (e.g. Courant \& Friedrichs 1948; Hornung 1986; Samtaney \& Pullin 1996 and originally Mach).

The development of computational algorithms for the simulation of gasdynamics problems has benefited greatly from use of so-called Riemann solvers (Roe 1981). In this method, local interpolation of the solution is based on a local physical solution to a one-dimensional unsteady gasdynamics problem rather than a non-physical highorder polynomial function. Direct solution of the one-dimensional unsteady Riemann problem is simplified by the fact that the equations describing the local solution remain hyperbolic for all initial conditions. Computational approaches that explicitly track the locations of strong shock waves have spurred great progress in the understanding of shock interactions since explicit solutions are now required for all situations of interactions between waves that might occur in the time evolution of the solution. In a frame of reference fixed to a shock intersection point, this requires the solution of a Riemann problem in two spatial dimensions only (not time). The problem in two spatial dimensions involves an additional difficulty caused by the possibility of mixed hyperbolic-elliptic equations with subsonic flow occurring in an arc about the intersection point. This complicates the geometrical construction of the local solution with $(p, \delta)$ plane methods because of the case distinctions that arise as the number of parameters is increased and the difficulty in determining the nature of the limiting cases at the boundaries of the interaction regimes. Further, since the solutions are synthesized arbitrarily in the $(p, \delta)$ plane, there is no understanding of the complete set of possible interactions (see Courant \& Friedrichs 1948 for discussion). Given this ambiguity, it has proved helpful to apply concepts from bifurcation theory in order to elucidate the non-uniqueness and non-existence problems that arise in the complex manifolds of possible solutions (see for example the review by Glimm 1988). The most complete description of the Riemann problem for gasdynamics in two spatial dimensions is that of Glimm et al. (1985) who unambiguously categorize the possible forms of two-dimensional steady-flow wave interactions based on certain physical assumptions and the fundamental solutions originally deduced by Meyer (1908). As discussed by Glimm et al. (1985), the solutions for the interaction problem in two spatial dimensions represent the elementary waves for the open two-dimensional Riemann problem (two spatial dimensions and time). Attempting now to put the current study in context, we formulate the problem in two spatial dimensions and time, but immediately reduce the problem to one angular dimension and time by a scaling argument. Consideration of steady-flow solutions reduces the problem to one angular dimension only and we obtain solutions largely equivalent to those of Glimm et al. (1985), but by novel means. Given the steady-flow solutions in one angular coordinate, we return briefly to consider the unsteady problem in one angular coordinate and time, and indicate a path that appears to hold promise for future investigation of the open problem of the stability of steady-flow shock-wave interactions. Finally, we note the relationship of the current study where we work in a cylindrically symmetric polar coordinate system to that of Taylor \& Maccoll (1933) who derive results in an axially symmetric polar coordinate system; in both cases, solutions are sought that are constant along a ray to reduce the problem to an o.d.e. in one angular coordinate.

The present study arises out of an experimental investigation of shock interference heating on blunt bodies at the GALCIT T5 hypervelocity shock tunnel (e.g. Sanderson 1995; Samtaney \& Meiron 1997; Sanderson, Hornung \& Sturtevant 2003). In order to determine the effect of caloric imperfections, the $(p, \delta)$ plane analysis method was 
extended to consider the chemically frozen and equilibrium limits of Lighthill's IDG model. The same difficulties of non-uniqueness and non-existence arise as discussed above. In both the frozen and equilibrium limits, the inviscid conservation equations retain the property of the Euler equations for flow of perfect gas whereby the equations are free of any fundamental length scale. In order to study chemical non-equilibrium effects on shock-wave interactions, whereby finite-rate chemical kinetics provide a fundamental length scale, it was proposed to construct a polar grid centred at the intersection point and solve numerically for the flow field. The interaction of the chemical scales and the fluid mechanical scales imposed by the external boundary conditions introduce phenomena that are not observed in perfect gas flows. The chemically frozen and equilibrium solutions obtained from the polar analysis form the boundary conditions for the computation at $r \rightarrow 0$ and $r \rightarrow \infty$, respectively. This leads to the formulation of conservation equations in $(r, \theta)$-coordinates. It is apparent that analytical treatment of the conservation equations in the $\theta$ direction in the limit $r \rightarrow 0$ might lead to further insight.

The analysis that follows is based on the hypothesis that the flow variables remain piecewise differentiable about the shock-wave intersection point. Given this assumption, it is possible to scale the conservation equations about the intersection point and seek local solutions that are constant along rays passing through the intersection point. The resulting local solutions therefore exhibit no length scale. Under this scaling, the conservation equations are shown to reduce exactly to a system of linear ordinary differential equations in one angular coordinate, $\theta$, about the intersection point. A simple analytical solution follows from the requirement for $2 \pi$ periodicity and the specification of an initial condition on the incoming streamline. This solution is simply the trivial case of uniform flow, and importantly, its uniqueness is conditional on the non-singularity of the Jacobian matrix giving the derivatives of the $\theta$-fluxes of the conserved quantities with respect to the primitive variables. The singularities so obtained may be reconciled with the conventional method-of-characteristics interpretation for non-homentropic flow. Each singularity of the Jacobian yields a family of solutions and further examination leads to a complete set of closed-form singular solutions. The central singular solution is familiar as a Prandtl-Meyer expansion and here a simple geometrical interpretation is obtained. Other singular behaviours are observed relating to the growth of the shear layer originating at shock intersection points. Weak solutions of the conservation equations may also be obtained under this approximation. This leads explicitly to the admissibility of oblique shock waves, shear layers and the inviscid wall-boundary condition as the complete set of solutions.

Solutions to standard shock-wave interaction problems may be obtained under certain additional assumptions. By imposing a condition of geometric regularity on the intersection of the incident waves and the usual entropy condition, solutions are formally restricted to not more than two incident rays and two outgoing waves. We go on to demonstrate the possible downstream matching conditions. With these restrictions formally established, it is possible to determine the complete set of locally scale-free solutions to the Euler equations about a point.

Much of this analysis reproduces results from classical gasdynamics in a different framework. The simple treatment and results offered here do not appear to be known in the literature. The justification for the current approach lies in the fact that the complete set of admissible solutions is obtained via a constructive process as the consequence of clearly defined assumptions. This rigour and completeness cannot be obtained from the conventional synthesis of solutions with polars in the pressure-flow 


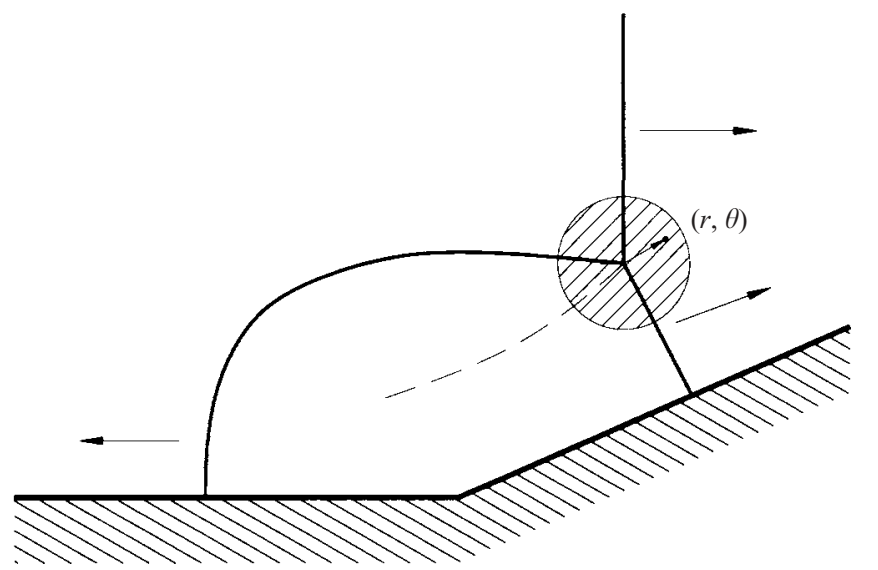

FIGURE 1. Region in vicinity of Mach reflection flow field that exhibits no length scale in the vicinity of the shock-wave intersection point.

deflection angle plane based on the fundamental wave solutions deduced by Meyer (1908).

\section{Solutions of the Euler equations about a point in cylindrical coordinates}

Applying Reynolds transport theorem to the principles of mass, momentum and energy conservation and employing the appropriate metrics for cylindrical coordinates we obtain the following differential equations for the conserved quantities:

$$
\begin{gathered}
\frac{\partial \rho}{\partial t}+\frac{1}{r} \frac{\partial\left(r \rho u_{r}\right)}{\partial r}+\frac{1}{r} \frac{\partial\left(\rho u_{\theta}\right)}{\partial \theta}=0 \\
\frac{\partial\left(\rho u_{r}\right)}{\partial t}+\frac{1}{r} \frac{\partial\left(r \rho u_{r}^{2}\right)}{\partial r}+\frac{1}{r} \frac{\partial\left(\rho u_{r} u_{\theta}\right)}{\partial \theta}-\frac{\rho u_{\theta}^{2}}{r}=-\frac{\partial p}{\partial r} \\
\frac{\partial\left(\rho u_{\theta}\right)}{\partial t}+\frac{1}{r} \frac{\partial\left(r \rho u_{r} u_{\theta}\right)}{\partial r}+\frac{1}{r} \frac{\partial\left(\rho u_{\theta}^{2}\right)}{\partial \theta}+\frac{\rho u_{r} u_{\theta}}{r}=-\frac{1}{r} \frac{\partial p}{\partial \theta}, \\
\frac{\partial\left(\rho e+\frac{1}{2} \rho u^{2}\right)}{\partial t}+\frac{1}{r} \frac{\partial\left[r\left(\rho e+\frac{1}{2} \rho u^{2}\right) u_{r}\right]}{\partial r}+\frac{1}{r} \frac{\partial\left[\left(\rho e+\frac{1}{2} \rho u^{2}\right) u_{\theta}\right]}{\partial \theta}+\frac{1}{r} \frac{\partial\left(r p u_{r}\right)}{\partial r}+\frac{1}{r} \frac{\partial\left(p u_{\theta}\right)}{\partial \theta}=0 .
\end{gathered}
$$

Here, $u^{2}=u_{r}^{2}+u_{\theta}^{2}, e=C_{v} T$ is the specific internal energy and the remaining symbols have their usual meanings.

In order to examine the possible local solutions in the immediate vicinity of the intersection point, we consider solutions where radial variations are sufficiently small that they may be neglected. That is, the $r$ derivatives of the dependent variables vanish. Such a reduction may always be obtained locally for radii that are small with respect to length scales imposed by the boundary conditions away from the intersection point with the additional assumption that the dependent variables remain piecewise differentiable (see figures 1 and 2). Equations (2.1)-(2.4) reduce to

$$
r \frac{\partial}{\partial t} \boldsymbol{A}(z)+\frac{\partial}{\partial \theta} \boldsymbol{B}(z)+\mathbf{C}(z)=0
$$




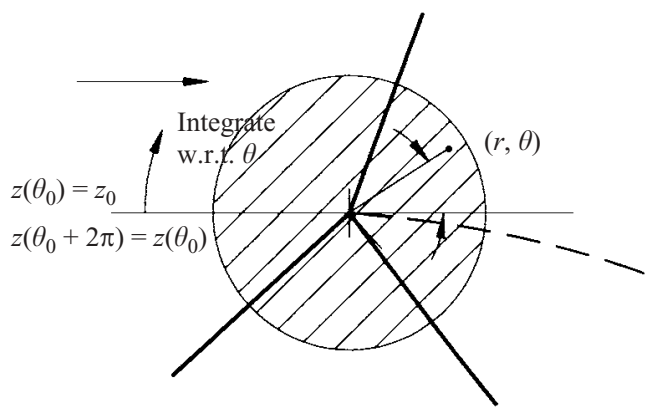

FIGURE 2. Integration of length-scale-free o.d.e.s about the shock intersection point with periodic boundary conditions.

where

$$
\boldsymbol{A}=\left[\begin{array}{c}
\rho \\
\rho u_{r} \\
\rho u_{\theta} \\
\rho e+\frac{1}{2} \rho u^{2}
\end{array}\right], \quad \boldsymbol{B}=\left[\begin{array}{c}
\rho u_{\theta} \\
\rho u_{r} u_{\theta} \\
\rho u_{\theta}^{2}+p \\
\left(\rho e+\frac{1}{2} \rho u^{2}+p\right) u_{\theta}
\end{array}\right], \quad \boldsymbol{C}=\left[\begin{array}{c}
\rho u_{r} \\
\rho\left(u_{r}^{2}-u_{\theta}^{2}\right) \\
2 \rho u_{r} u_{\theta} \\
\left(\rho e+\frac{1}{2} \rho u^{2}+p\right) u_{r}
\end{array}\right] .
$$

Here, for a perfect gas, $\rho e=p /(\gamma-1)$ and

$$
z=\left[\begin{array}{c}
\rho \\
u_{r} \\
u_{\theta} \\
p
\end{array}\right]
$$

Consider now steady solutions of this system of equations

$$
\frac{\mathrm{d}}{\mathrm{d} \theta} \boldsymbol{B}(z)+\boldsymbol{C}(z)=0
$$

with

$$
\begin{gathered}
z\left(\theta_{0}\right)=z_{0}, \\
z\left(\theta_{0}+2 \pi\right)=\boldsymbol{z}\left(\theta_{0}\right) .
\end{gathered}
$$

This set of o.d.e.s giving the equilibrium solutions can be integrated (in the sense of classical solutions) with respect to $\theta$ from an appropriate free-stream boundary condition (see figure 2). The issue of shock waves and other weak solutions will be addressed shortly.

Forming the Jacobian matrix, $\boldsymbol{B}_{z}$, giving the derivatives of the $\theta$-fluxes with respect to the primitive variables, $z,(2.8)$ may be expressed in normal form

$$
\frac{\mathrm{d} B(z)}{\mathrm{d} z} \frac{\mathrm{d} z}{\mathrm{~d} \theta}+\boldsymbol{C}(z)=0 .
$$

For the given fluxes, it follows that

$$
\boldsymbol{B}_{z}=\left[\begin{array}{cccc}
u_{\theta} & 0 & \rho & 0 \\
u_{r} u_{\theta} & \rho u_{\theta} & \rho u_{r} & 0 \\
u_{\theta}^{2} & 0 & 2 \rho u_{\theta} & 1 \\
\frac{1}{2} u_{\theta} u^{2} & \rho u_{r} u_{\theta} & \frac{\gamma p}{\gamma-1}+\frac{1}{2} \rho u^{2}+\rho u_{\theta}^{2} & \frac{\gamma u_{\theta}}{\gamma-1}
\end{array}\right]
$$




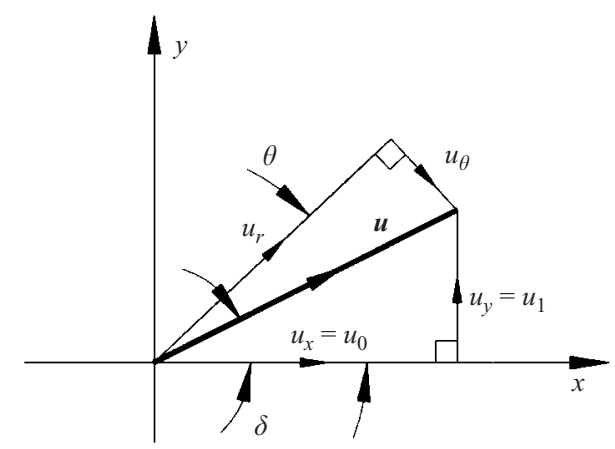

FIGURE 3. Interpretation of solution as a constant thermodynamic state with velocity components varying according to a rotational transformation about the intersection point.

and after some manipulation it is possible to show that

$$
\frac{\mathrm{d} z}{\mathrm{~d} \theta}=\frac{\mathrm{d}}{\mathrm{d} \theta}\left[\begin{array}{c}
\rho \\
u_{r} \\
u_{\theta} \\
p
\end{array}\right]=-\boldsymbol{B}_{z}^{-1} \boldsymbol{C}=\left[\begin{array}{c}
0 \\
u_{\theta} \\
-u_{r} \\
0
\end{array}\right], \quad\left|\boldsymbol{B}_{z}\right| \neq 0 .
$$

The thermodynamic variables separate from the velocity components in the solution and are constant with respect to $\theta$. Eliminating $u_{\theta}$, it follows that

$$
\frac{\mathrm{d}^{2} u_{r}}{\mathrm{~d} \theta^{2}}+u_{r}=0
$$

with general solutions

$$
\begin{gathered}
u_{r}=u_{0} \cos \theta+u_{1} \sin \theta, \\
u_{\theta}=\frac{\mathrm{d} u_{r}}{\mathrm{~d} \theta}=-u_{0} \sin \theta+u_{1} \cos \theta
\end{gathered}
$$

We identify this form as a coordinate rotation through an angle $\theta$ and so $u_{0}$ and $u_{1}$ are identifiable as the Cartesian $x$ and $y$ components, respectively, of the velocity vector. With respect to the local flow deflection angle, $\delta$, we therefore have

$$
\left[\begin{array}{l}
u_{x} \\
u_{y}
\end{array}\right]=\left[\begin{array}{l}
u_{0} \\
u_{1}
\end{array}\right]=\left[\begin{array}{l}
u \cos \delta \\
u \sin \delta
\end{array}\right]
$$

and it follows that

$$
\left[\begin{array}{l}
u_{r} \\
u_{\theta}
\end{array}\right]=u\left[\begin{array}{cc}
\cos \theta & \sin \theta \\
-\sin \theta & \cos \theta
\end{array}\right]\left[\begin{array}{c}
\cos \delta \\
\sin \delta
\end{array}\right]=u\left[\begin{array}{c}
\cos (\theta-\delta) \\
-\sin (\theta-\delta)
\end{array}\right] .
$$

The solution we have obtained is that of completely uniform flow in the vicinity of the point. The solution represents a constant thermodynamic state with respect to $\theta$ (i.e. $p=$ constant, $\rho=$ constant) and the velocity components vary according to the angle of rotation of the coordinate system about the origin (see figure 3 ). This great simplification should come as no surprise given the original hypothesis of solutions without a length scale. 


\section{Singular solutions}

Having dealt with the regular solution we now turn to the more interesting cases when the solution obtained is not unique, that is, when $\left|\boldsymbol{B}_{z}\right|=0$. In fact

$$
\left|\boldsymbol{B}_{z}\right|=\frac{\rho u_{\theta}^{2}}{\gamma-1}\left(\gamma p-\rho u_{\theta}^{2}\right) .
$$

Note that $\left|\boldsymbol{B}_{z}\right| \sim M_{\theta}^{2}\left(1-M_{\theta}^{2}\right)$; however, the dimensional form retains important cases where $\rho, p \rightarrow 0$. Three cases arise such that $\left|\boldsymbol{B}_{z}\right|=0$ :

(a) $p=\rho u_{\theta}^{2} / \gamma$; this corresponds to a Mach line where $M_{\theta}^{2}=1$.

(b) $u_{\theta}^{2}=0$; this double root corresponds to the convective streamline passing through the intersection point.

(c) $\rho=0$; this root is identified with the situation of maximum turning to a vacuum through a Prandtl-Meyer expansion.

In what follows, we introduce the notation $M_{\theta}=u_{\theta} / a$ and $M_{r}=u_{r} / a$ for the velocity components normalized with respect to the local speed of sound. It is meaningful to describe the components of a Mach number in this way since it is well known that the Rankine-Hugoniot conditions for oblique shock waves are identical to those for a normal shock provided that velocity components normal to the wavefront are employed. Further, we allow $M_{\theta}$ and $M_{r}$ to take negative values, as required by the sign of the respective velocity components in the current coordinate system. Although unconventional, this is required to preserve the generality of the formulation and poses no difficulty. Note that the characteristic directions obtained from method-ofcharacteristics analysis for the general case of non-homentropic flow are reflected in the singularities of $\boldsymbol{B}_{z}$. The singularities $M_{\theta}^{2}=1$ correspond to the acoustic characteristics that propagate the Riemann invariants. The streamline singularities, $u_{\theta}^{2}=0$, propagate the entropy and stagnation enthalpy invariants.

\subsection{Singular solutions for $M_{\theta}^{2}=1$}

Consider initially the family of singular solutions that arise in the case $M_{\theta}^{2}=1$. Retaining only the first three of (2.8) and eliminating $p$ according to $p=\rho u_{\theta}^{2} / \gamma$ we have the reduced system of equations

$$
\frac{\mathrm{d}}{\mathrm{d} \theta} \boldsymbol{B}_{1}\left(z_{1}\right)+\boldsymbol{C}_{1}\left(z_{1}\right)=0 ; \quad \boldsymbol{B}_{1}=\left[\begin{array}{c}
\rho u_{\theta} \\
\rho u_{r} u_{\theta} \\
\frac{\gamma+1}{\gamma} \rho u_{\theta}^{2}
\end{array}\right], \quad \boldsymbol{C}_{1}=\left[\begin{array}{c}
\rho u_{r} \\
\rho\left(u_{r}^{2}-u_{\theta}^{2}\right) \\
2 \rho u_{r} u_{\theta}
\end{array}\right] .
$$

Again, after some manipulation we obtain

$$
\frac{\mathrm{d} z_{1}}{\mathrm{~d} \theta}=\frac{\mathrm{d}}{\mathrm{d} \theta}\left[\begin{array}{c}
\rho \\
u_{r} \\
u_{\theta}
\end{array}\right]=-\boldsymbol{B}_{1 z_{1}}{ }^{-1} \boldsymbol{C}_{1}=\left[\begin{array}{c}
\frac{-2}{\gamma+1} \frac{\rho u_{r}}{u_{\theta}} \\
u_{\theta} \\
-\frac{\gamma-1}{\gamma+1} u_{r}
\end{array}\right] ; \quad\left|\boldsymbol{B}_{1 z_{1}}\right|=\frac{\gamma+1}{\gamma} \rho^{2} u_{\theta}^{3} \neq 0 .
$$

Again the velocity components decouple from the thermodynamic quantities. Define

$$
\Gamma^{2}=\frac{\gamma-1}{\gamma+1} .
$$


Eliminating $u_{\theta}$ between the second and third of (3.3) it follows that

$$
\frac{\mathrm{d}^{2} u_{r}}{\mathrm{~d} \theta^{2}}+\Gamma^{2} u_{r}=0
$$

with general solution

$$
u_{r}=u_{0} \cos (\Gamma \theta)+u_{1} \sin (\Gamma \theta) .
$$

The arbitrary coefficients $u_{0}$ and $u_{1}$ are distinct from those introduced previously. It follows immediately that

$$
u_{\theta}=-u_{0} \Gamma \sin (\Gamma \theta)+u_{1} \Gamma \cos (\Gamma \theta) .
$$

Multiplying $u_{r}$ by $\Gamma$ and expressing in matrix form:

$$
\left[\begin{array}{c}
\Gamma u_{r} \\
u_{\theta}
\end{array}\right]=\left[\begin{array}{cc}
\cos (\Gamma \theta) & \sin (\Gamma \theta) \\
-\sin (\Gamma \theta) & \cos (\Gamma \theta)
\end{array}\right]\left[\begin{array}{c}
\Gamma u_{0} \\
\Gamma u_{1}
\end{array}\right]
$$

Inverting this expression we can determine the unknown coefficients, $u_{0}$ and $u_{1}$, according to the conditions existing at some point $\theta=\theta_{1}$ within the singular region. With the coefficients thus determined, we then have the solution for all other points $\theta=\theta_{2}$ within the singular region. After manipulation we have;

$$
\left[\begin{array}{c}
\Gamma u_{r_{2}} \\
u_{\theta_{2}}
\end{array}\right]=\left[\begin{array}{cc}
\cos \left[\Gamma\left(\theta_{2}-\theta_{1}\right)\right] & \sin \left[\Gamma\left(\theta_{2}-\theta_{1}\right)\right] \\
-\sin \left[\Gamma\left(\theta_{2}-\theta_{1}\right)\right] & \cos \left[\Gamma\left(\theta_{2}-\theta_{1}\right)\right] .
\end{array}\right]\left[\begin{array}{c}
\Gamma u_{r_{1}} \\
u_{\theta_{1}}
\end{array}\right] .
$$

Thus, (3.9) relates all points within the singular region, including the special case where $\theta_{1}$ and $\theta_{2}$ are taken to be the upstream and downstream boundaries of the singular region. We see that the family of singular solutions for $M_{\theta}^{2}=1$ may be interpreted as an anisotropic stretching operation in the radial direction, followed by a rotation operation through angle $\sqrt{(\gamma-1) /(\gamma+1)}\left(\theta_{2}-\theta_{1}\right)$ and a complementary anisotropic stretching in the rotated frame. Non-dimensionalizing by $u_{\theta_{1}}$ and since $u_{r_{1}} / u_{\theta_{1}}=$ $\cot \left(\delta_{1}-\theta_{1}\right)$ (see figure 3 ):

$$
\left[\begin{array}{cc}
\left(\Gamma \cot \left(\delta_{1}-\theta_{1}\right)\right) \frac{u_{r_{2}}}{u_{r_{1}}} \\
\frac{u_{\theta_{2}}}{u_{\theta_{1}}}
\end{array}\right]=\left[\begin{array}{cc}
\cos \left[\Gamma\left(\theta_{2}-\theta_{1}\right)\right] & \sin \left[\Gamma\left(\theta_{2}-\theta_{1}\right)\right] \\
-\sin \left[\Gamma\left(\theta_{2}-\theta_{1}\right)\right] & \cos \left[\Gamma\left(\theta_{2}-\theta_{1}\right)\right] .
\end{array}\right]\left[\begin{array}{c}
\Gamma \cot \left(\delta_{1}-\theta_{1}\right) \\
1
\end{array}\right] .
$$

Thus, the radial and tangential velocity ratios are parameterized by the single variable $\theta_{2}$ for given upstream conditions, $\theta_{1}$ and $\delta_{1}$.

It remains to integrate the first of (3.3). A more convenient approach is to observe that $M_{\theta}^{2}=1$, or $M_{\theta}= \pm 1$, for all $\theta$ in the singular family of solutions and to express the remaining quantities in term of the known values of $u_{\theta_{2}} / u_{\theta_{1}}$ and $u_{r_{2}} / u_{r_{1}}$ so that

$$
\frac{a_{2}}{a_{1}}=\frac{a_{2}}{u_{\theta_{2}}} \frac{u_{\theta_{2}}}{u_{\theta_{1}}} \frac{u_{\theta_{1}}}{a_{1}}=\frac{M_{\theta_{1}}}{M_{\theta_{2}}} \frac{u_{\theta_{2}}}{u_{\theta_{1}}}=\frac{u_{\theta_{2}}}{u_{\theta_{1}}} .
$$

Since adiabatic reversible flow is generally isentropic, then for a perfect gas we have

$$
\frac{p_{2}}{p_{1}}=\left(\frac{\rho_{2}}{\rho_{1}}\right)^{\gamma}=\left(\frac{a_{2}}{a_{1}}\right)^{2 \gamma /(\gamma-1)}=\left(\frac{u_{\theta_{2}}}{u_{\theta_{1}}}\right)^{2 \gamma /(\gamma-1)} .
$$

Now since $M^{2}=M_{\theta}^{2}+M_{r}^{2}=1+M_{r}^{2}$ throughout the singular region, we have

$$
M_{2}^{2}=1+M_{r_{2}}^{2}=1+\left(M_{r_{1}} \frac{u_{r_{2}} a_{1}}{u_{r_{1}} a_{2}}\right)^{2}=1+\left(\frac{u_{r_{2}}}{u_{r_{1}}}\right)^{2}\left(\frac{u_{\theta_{1}}}{u_{\theta_{2}}}\right)^{2}\left(M_{1}^{2}-1\right) .
$$


Finally, the downstream flow deflection angle is determined from

$$
\cot \left(\delta_{2}-\theta_{2}\right)=\frac{u_{r_{2}}}{u_{\theta_{2}}}=\frac{u_{r_{2}}}{u_{r_{1}}} \frac{u_{r_{1}}}{u_{\theta_{1}}} \frac{u_{\theta_{1}}}{u_{\theta_{2}}}=\frac{u_{r_{2}}}{u_{r_{1}}} \frac{u_{\theta_{1}}}{u_{\theta_{2}}} \cot \left(\delta_{1}-\theta_{1}\right) .
$$

The above family of solutions is in fact synonymous with that obtained by Prandt and Meyer for an expansion fan acting over local Mach angles $\theta_{1}$ to $\theta_{2}$. The singular cases when $\left|\boldsymbol{B}_{1 z_{1}}\right|=((\gamma+1) / \gamma) \rho^{2} u_{\theta}^{3}=0$ are special cases of the singularities of the original equations and these are treated in the following sections.

\subsection{Singular solutions for $u_{\theta}^{2}=0$}

We identify the case $u_{\theta}^{2}=0$ with the two characteristic directions that typically arise along the streamline direction. For non-homentropic flow, the invariant quantities along the two streamline oriented characteristics are entropy and stagnation enthalpy. In the case of $u_{\theta}^{2}=0$, the Jacobian, $\boldsymbol{B}_{z}$, reduces to

$$
\boldsymbol{B}_{2 z}=\left[\begin{array}{cccc}
0 & 0 & \rho & 0 \\
0 & 0 & \rho u_{r} & 0 \\
0 & 0 & 0 & 1 \\
0 & 0 & \frac{\gamma p}{\gamma-1}+\frac{1}{2} \rho u_{r}^{2} & 0
\end{array}\right], \quad \boldsymbol{c}_{2}=\left[\begin{array}{c}
\rho u_{r} \\
\rho u_{r}^{2} \\
0 \\
\left(\frac{\gamma p}{\gamma-1}+\frac{1}{2} \rho u_{r}^{2}\right) u_{r}
\end{array}\right],
$$

such that

$$
\frac{\mathrm{d} \boldsymbol{B}_{2}(z)}{\mathrm{d} z} \frac{\mathrm{d} z}{\mathrm{~d} \theta}+\boldsymbol{C}_{2}(z)=0 .
$$

From the first of these equations, it immediately follows that

$$
\rho u_{r}=0
$$

and from the third that

$$
\frac{\partial p}{\partial \theta}=0
$$

Excluding for the moment the case $\rho=0$, which will be discussed in the following section, we are left with the family of singular solutions $u_{r}=0, u_{\theta}=0, p(\theta)=$ constant, and $\rho(\theta)$ remains undetermined. Physically, this solution represents a nonhomenthalpic stagnation point where the density and hence temperature and enthalpy are allowed to vary arbitrarily with $\theta$.

\subsection{Singular solutions for $\rho=0$}

In the case of $\rho=0$, the Jacobian, $\boldsymbol{B}_{z}$, reduces to

$$
\boldsymbol{B}_{3 z}=\left[\begin{array}{cccc}
u_{\theta} & 0 & 0 & 0 \\
u_{r} u_{\theta} & 0 & 0 & 0 \\
u_{\theta}^{2} & 0 & 0 & 1 \\
\frac{1}{2} u_{\theta} u^{2} & 0 & \frac{\gamma p}{\gamma-1} & \frac{\gamma u_{\theta}}{\gamma-1}
\end{array}\right], \quad \boldsymbol{c}_{3}=\left[\begin{array}{c}
0 \\
0 \\
0 \\
\frac{\gamma p}{\gamma-1} u_{r}
\end{array}\right]
$$

such that

$$
\frac{\mathrm{d} \boldsymbol{B}_{3}(z)}{\mathrm{d} z} \frac{\mathrm{d} z}{\mathrm{~d} \theta}+\boldsymbol{C}_{3}(z)=0 .
$$

The first two of these equations are trivially satisfied under the constraint $\rho=0$. The fourth equation is also trivially satisfied since $p=\rho R T=0$. The third remaining 
equation reduces to

$$
\frac{\partial p}{\partial \theta}=0
$$

or $p=$ constant. Again, we find that $p=\rho R T=0$. We are left with the family of singular solutions $\rho=0, p=0$ with $u_{r}(\theta)$ and $u_{\theta}(\theta)$ remaining undetermined. Physically, this solution represents the state obtained after expansion to a vacuum.

\section{Weak solutions of the Euler equations about a point}

Having examined the regular and singular solutions admitted by the Euler equations about a point, we now consider weak solutions of the conservation equations (2.5) under the original assumptions that the local solutions lack a length scale and that the dependent variables remain piecewise differentiable. We seek weak solutions that satisfy the conservation equations in the integral sense of

$$
\lim _{\zeta \rightarrow 0}\left[r \int_{\beta-\zeta}^{\beta+\zeta} \frac{\partial}{\partial t} \boldsymbol{A}(\boldsymbol{z}) \mathrm{d} \theta+\int_{\beta-\zeta}^{\beta+\zeta} \frac{\partial}{\partial \theta} \boldsymbol{B}(z) \mathrm{d} \theta+\int_{\beta-\zeta}^{\beta+\zeta} \boldsymbol{C}(z) \mathrm{d} \theta\right]=0 .
$$

By the Leibniz rule,

$$
r \frac{\partial}{\partial t} \int_{\beta^{-}}^{\beta^{+}} \boldsymbol{A} \mathrm{d} \theta-\left(\boldsymbol{A}\left(\beta^{+}\right) \dot{\beta}-\boldsymbol{A}\left(\beta^{-}\right) \dot{\beta}\right) r+\left(\boldsymbol{B}\left(\beta^{+}\right)-\boldsymbol{B}\left(\beta^{-}\right)+0=0 .\right.
$$

Here, $\dot{\beta}=\partial \beta / \partial t$ and $\beta^{ \pm}=\lim _{\zeta \rightarrow 0} \beta \pm \zeta$. The first integral tends to zero in the limit $\zeta \rightarrow 0$ when $\boldsymbol{A}$ remains finite. We are left with

$$
-r \dot{\beta} \Delta[\boldsymbol{A}]+\Delta[\boldsymbol{B}]=0
$$

where $\Delta[\cdot]$ represents the jump in the prescribed quantity at location $\beta$. Consider now the steady solutions

$$
\Delta[\boldsymbol{B}]=\Delta\left[\begin{array}{c}
\rho u_{\theta} \\
\rho u_{r} u_{\theta} \\
\rho u_{\theta}^{2}+p \\
\left(\frac{\gamma p}{\gamma-1}+\frac{1}{2} \rho u^{2}\right) u_{\theta}
\end{array}\right]=0 .
$$

From the first of these it follows that

$$
\frac{\rho_{2} u_{\theta_{2}}}{\rho_{1} u_{\theta_{1}}}=1
$$

and from the second that

$$
u_{r_{1}}=\frac{\rho_{2} u_{\theta_{2}}}{\rho_{1} u_{\theta_{1}}} u_{r_{2}}=u_{r_{2}}, \quad \rho_{1} u_{\theta_{1}} \neq 0
$$

We must therefore consider the regular case whereby the conservation of both mass and radial momentum determine that $\Delta\left[u_{r}\right]=0$, and the singular case whereby $\rho_{1} u_{\theta_{1}}=0$. 
4.1. Regular weak solutions

In the regular case considered above, $\Delta\left[u_{r}\right]=0$, the remaining equations reduce directly to:

$$
\Delta\left[\begin{array}{c}
\rho u_{\theta} \\
\rho u_{\theta}^{2}+p \\
\left(\frac{\gamma p}{\gamma-1}+\frac{1}{2} \rho u^{2}\right) u_{\theta}
\end{array}\right]=0
$$

with solutions:

$$
\begin{gathered}
\frac{\rho_{2}}{\rho_{1}}=\left\{\begin{array}{l}
1, \\
\frac{(\gamma+1) M_{\theta_{1}}^{2}}{2+(\gamma-1) M_{\theta_{1}}^{2}},
\end{array}\right. \\
\frac{p_{2}}{p_{1}}=\left\{\begin{array}{l}
1, \\
\frac{1+\gamma\left(2 M_{\theta_{1}}^{2}-1\right)}{\gamma+1}, \\
\frac{u_{\theta_{2}}}{u_{\theta_{1}}}=\frac{\rho_{1}}{\rho_{2}} .
\end{array}\right.
\end{gathered}
$$

Clearly, these solutions reproduce the well-known behaviour of oblique shock waves whereby the solutions are obtained using components normal to the shock front. Further, it is known that only waves with $M_{\theta_{1}}^{2}>1$ are entropy producing with $M_{\theta_{2}}^{2}<1$.

\subsection{Irregular weak solutions}

In the irregular case considered above, $\rho_{1} u_{\theta_{1}}=0$, the equations reduce to;

$$
\Delta[\boldsymbol{B}]=\Delta\left[\begin{array}{c}
p \\
p u_{\theta}
\end{array}\right]=0 .
$$

Excluding the exceptional case $\rho_{1}=0$ then $p_{2}=p_{1}, u_{\theta_{2}}=u_{\theta_{1}}=0$ and $\Delta[\rho]$ and $\Delta\left[u_{r}\right]$ remain undetermined. This formally admits a shear layer as a weak solution of the Euler equations about a point. In the further degenerate case, $u_{r_{2}}=0$, we have described a wall boundary condition such as occurs in the case of flow over a wedge or the free streamline boundary that occurs in the expansion of a supersonic jet. The exceptional case, $\rho_{1}=0$, arises when the shear layer or wall coincides with a turning expansion of the flow to a vacuum.

\section{Synthesis of solutions}

At this point, we have studied the solutions of the Euler equations about a point and have examined the set of solutions that may arise. Specifically we find:

(a) A regular solution that corresponds to uniform flow.

(b) Families of singular solutions that arise,

when $M_{\theta}^{2}=1$, when $u_{\theta}^{2}=0$, when $\rho=0$.

(c) Families of weak solutions that satisfy the Rankine-Hugoniot relations for oblique shock waves, that arise when $\rho u_{\theta}=0$ and admit shear layers and wall boundary conditions. These solutions have been obtained via a constructive process from the two-dimensional Euler equations and follow from the simple assumptions that the local flow field 
(a)

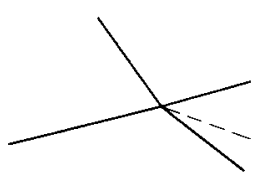

(d)

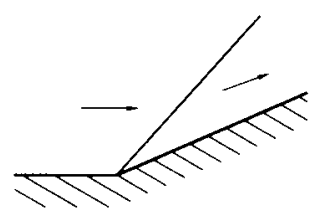

(g)

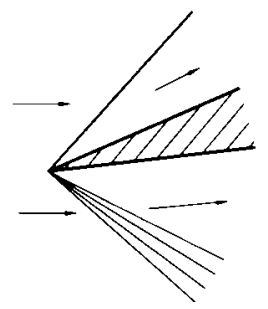

(j)

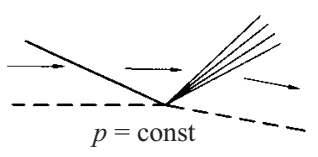

(b)

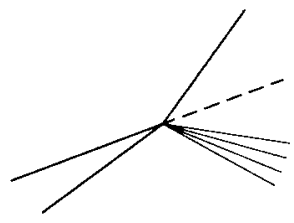

(e)

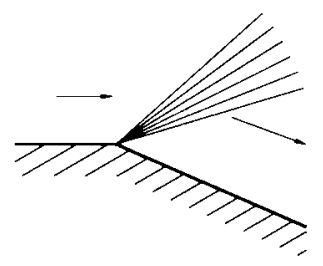

(h)

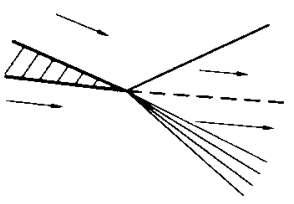

(k)

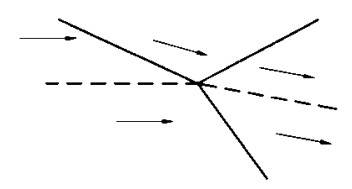

(c)

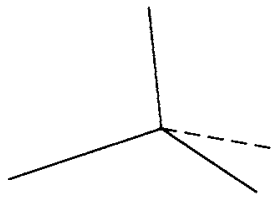

(f)

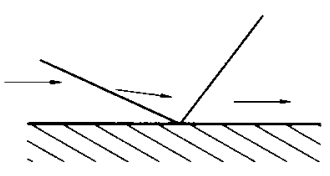

(i)

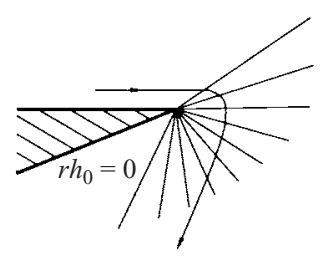

(l)

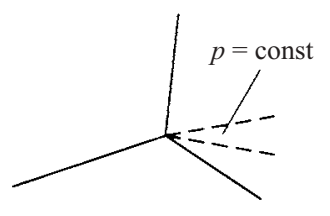

FIGURE 4. Unification of classical gasdynamics problems.

exhibits no length scale and remains piecewise differentiable. It comes as no surprise therefore that this formulation appears to unify many of the classical problems of gasdynamics (see figure 4). In the context of Glimm et al. (1985), we have obtained a complete set of elementary waves in one angular coordinate. To address the further goal of defining a complete set of wave interactions in one angular coordinate, additional development is required and this is elaborated in the following sections.

\subsection{Prototypical interaction problem}

Generally, we see that complex wave-interaction configurations can be represented within the current framework (see figure 5). We will use the configuration shown in figure 5 as a specific example of the problems that arise in attempting to integrate the equations for shock-wave-interaction problems. Our goal here is to use the properties derived for the elementary waves in the previous sections to restrict the space of possible solutions, leaving only the set of allowable wave interactions. 


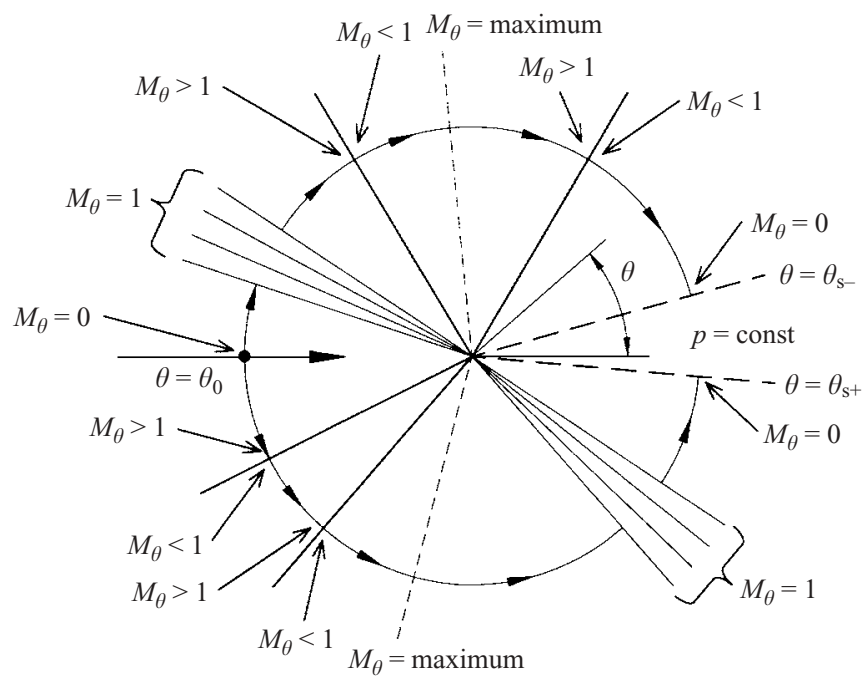

FIGURE 5. Synthesis of wave interaction solutions from elementary waves.

Recall that (2.13) are singular for $u_{\theta}=0$ and that the weak shear-layer solution that applies when $u_{\theta}=0$ (see $\left.\S 4.2\right)$ is inadequate for propagating all of the flow variables across the boundary. Under no circumstances can the solution therefore be continued across a ray where $u_{\theta}=0$. We therefore begin the solution from either side of the incident streamline at $\theta_{0}^{ \pm}=\left[\pi-\delta_{0}\right]^{ \pm}$where the free-stream conditions are specified at the upstream flow-deflection angle, $\delta_{0}$. Integrating from $\theta=\theta_{0}^{-}$in the $-\theta$-direction, the tangential Mach number, $\left|M_{\theta}\right|$, increases from the initial value of zero. Eventually $\left|M_{\theta}\right|=1$ and the system of equations becomes singular as discussed previously. At this point we choose to insert a singular compression fan and maintain $\left|M_{\theta}\right|=1$ over a region of arbitrary angle, as determined by hypothetical upstream boundary conditions. Exiting the singular region, $\left|M_{\theta}\right|$ continues to increase so that $\left|M_{\theta}\right|>1$. Again based on hypothetical upstream boundary conditions we choose to insert a shock wave and so downstream of the shock wave $\left|M_{\theta}\right|<1$ (see $\S 4$.1). Continuing the integration, $\left|M_{\theta}\right|$ continues to increase until a maximum is reached when the ray is perpendicular to the local streamline direction. Observe from (2.18) that

$$
\frac{\mathrm{d} M_{\theta}}{\mathrm{d} \theta}=\frac{\mathrm{d}}{\mathrm{d} \theta}\left(\frac{u_{\theta}}{a}\right)=\frac{-u}{a} \cos (\theta-\delta)
$$

and so $\left|M_{\theta}\right|$ has a maximum when $\theta-\delta=(n+1 / 2) \pi$. The points where $\theta-\delta=$ $(n+1 / 2) \pi$ also delineate the incident $\left(M_{r}<0,|\theta-\delta|>\pi / 2\right)$ and reflected $\left(M_{r}>0\right.$, $|\theta-\delta|<\pi / 2)$ wave regions, since (see (2.18))

$$
M_{r}=\frac{u_{r}}{a}=\frac{u}{a} \cos (\theta-\delta) .
$$

Beyond the point $|\theta-\delta|=\pi / 2,\left|M_{\theta}\right|$ decreases and at some point where $\left|M_{\theta}\right|>1$ we insert an oblique shock wave so that the tangential Mach number becomes subsonic, $\left|M_{\theta}\right|<1$. The integration continues with $\left|M_{\theta}\right|$ decreasing until we reach the point, $\theta=\theta_{s-}$, that cannot be crossed since $\left|M_{\theta}\right| \rightarrow 0$. Similar arguments apply when integrating from $\theta=\theta_{0}^{+}$towards $\theta=\theta_{s+}$ in the $+\theta$-direction. Eventually, a ray is reached where $\left|M_{\theta}\right| \rightarrow 0$ and again the solution cannot be continued. The incident waves shown in figure 5 where $M_{r}<0$ (shocks, fans, shear layers) must be specified 
on the basis of upstream conditions and the parameters for the reflected waves with $M_{r}>0$ are determined by the matching conditions at the two downstream rays where $M_{\theta}=0$.

\subsection{Restrictions on incident wave configurations}

To this point, no constraint has been placed on the number of incident and reflected waves and clearly we must introduce additional constraints to obtain the set of physically meaningful solutions. We hypothesize that the following conditions produce the desired set:

(a) Coincidence condition I: interaction configurations with a total of more than two regular or irregular weak elementary waves (i.e. shock waves or shear layers) in the incident wave region are disallowed.

(b) Coincidence condition II: singular regions with $\left|M_{\theta}\right|=1$ are disallowed in the incident wave region (i.e. Prandtl-Meyer compression waves).

(c) Entropy condition: elementary waves that reduce entropy in the direction of flow are disallowed (i.e. expansion shock waves).

Coincidence conditions I and II ensure that no more than two rays are required to meet at a point, as is apparent from inspection of figure 5. Contrary configurations require more than two rays to meet at a point, a requirement that is geometrically irregular and therefore physically irrelevant. The entropy condition reflects the usual result that for all entropy producing compression shocks, $\left|M_{\theta_{1}}\right|>1$ and $\left|M_{\theta_{2}}\right|<1$ (see $\S 4.1)$.

\subsection{Set of allowable incident-wave configurations}

Considering the possible elementary waves listed at the beginning of $\S 5$, subject to the conditions of $\S 5.2$, we may logically tabulate the complete set of allowable incident wave configurations according to the total number of waves:

(a) Zero incident waves:

a region of uniform flow,

a singular region with $u_{\theta}^{2}=0$ throughout,

a singular region with $\rho=0$ throughout.

(b) One incident wave:

a single incident entropy producing shock wave, a single incident shear layer.

(c) Two incident waves:

two incident entropy producing shock waves (of opposite sign, or overtaking with same sign (see figures $4 a$ and $4 b$ ),

one incident entropy producing shock wave and one incident shear layer, two incident shear layers (adjoined by a singular solution according to § 3.2),

(d) Configurations with more than two incident waves are geometrically irregular. The solutions over the arcs adjoining the incident waves and throughout the incident wave region downstream of the incident waves are limited to the remaining solutions listed at the beginning of $\S 5$ :

regions of uniform flow,

singular regions with $u_{\theta}^{2}=0$ throughout, or singular regions with $\rho=0$ throughout.

This represents a complete set of the possible incident wave configurations based on the allowable elementary waves. Figure 4 represents a subset of this complete set, drawn from flows that are familiar in classical gasdynamics. The third sub-case of $(c)$ above is unconventional but is admitted by the formulation and the imposed 
constraints, it is familiar as the flow that occurs at the downstream end of a wake flow, in the inviscid limit.

Additional solutions are possible (e.g. figures $4 d-4 i$ ) under the assumption that the physical solution over certain regions are described by a different set of equations, e.g. the equations of elasticity for the behaviour of a solid at the shock intersection point. As before, the constraint of geometric regularity on the intersection configuration requires that boundary conditions be imposed on no more than two rays passing through the origin.

\subsection{Entropy condition limits number of transmitted waves}

The entropy condition of the previous section eliminates incident expansion shock waves from consideration and may also be shown to concomitantly restrict the number of reflected waves upstream of the rays where the $M_{\theta}=0$ singularities occur to no more than one per side.

We have previously noted that for entropy producing compression shocks, $\left|M_{\theta_{1}}\right|>1$ and $\left|M_{\theta_{2}}\right|<1$. Note also that for expansion fans $\left|M_{\theta}\right|=1$ throughout the singular region. Finally, for any constant state zones in the reflected wave region, $|\theta-\delta|<\pi / 2$, we have $\left|M_{\theta}\right|$ decreasing in the downstream direction (see (5.1)). Therefore across the entire reflected wave region comprising shocks, fans and constant state regions, $\left|M_{\theta}\right|$ is decreasing or constant in the downstream direction.

If $\left|M_{\theta}\right| \geqslant 1$ entering the reflected wave region, then one reflected compression shock excludes the presence of another since $\left|M_{\theta_{2}}\right|<1$ for the first shock and $\left|M_{\theta_{1}}\right|>1$ is impossible for the second shock since $\left|M_{\theta}\right|$ is always decreasing or constant. It is impossible for a fan with $\left|M_{\theta}=1\right|$ to exist upstream of a shock since we require $\left|M_{\theta_{1}}\right|>1$ for the shock and $\left|M_{\theta}\right|$ must increase upstream of the shock. It is impossible for a fan with $\left|M_{\theta}=1\right|$ to exist downstream of a shock since we require $\left|M_{\theta_{2}}\right|<1$ for the shock and $\left|M_{\theta}\right|$ must decrease downstream of the shock. Two fans are excluded since they, in fact, represent a single contiguous fan with $\left|M_{\theta}=1\right|$ throughout.

If $\left|M_{\theta}\right|<1$ entering the reflected wave region, then no reflected waves or fans are possible since $\left|M_{\theta}\right|$ is decreasing or constant in the downstream direction.

Therefore no more than one reflected shock or reflected fan is possible per side in reflected wave region $|\theta-\delta|<\pi / 2$.

\subsection{Matching conditions at downstream singularities}

Having limited the number of incident and reflected waves to a finite number (no more than 2 incident, 2 reflected), it remains to determine the allowable matching conditions across the singularities that occur at $\theta=\theta_{s \pm}$ (see figure 5). Conventionally, this requires that the pressure and flow deflection angle be matched across a shear layer adjoining the two downstream rays where $M_{\theta}=0$. Alternatively, the $M_{\theta}=0$ rays may remain distinct by inserting two shear layers and a region of constant pressure (a singular solution of the type discussed in $\$ 3.2$ ) as discussed previously by Liepmann (B. Sturtevant 1991, personal communication) (see figure $4 l$ ). These matching conditions arise rigorously from the current formulation.

\subsubsection{Formal matching conditions}

We have shown in $\S 5.4$ that, at most, one reflected wave is allowable upstream of each of $\theta=\theta_{s \pm}$. Downstream of these reflected waves $\left|M_{\theta}\right| \leqslant 1$ and we have demonstrated that $\left|M_{\theta}\right|$ is decreasing in the downstream direction. The only remaining solutions upstream of the $\theta=\theta_{s \pm}$ singularities are therefore constant-state solutions, and the uniqueness of each is assured since $\left|\boldsymbol{B}_{z}\right| \neq 0$. Therefore, we must eventually obtain $u_{\theta}=0$ at the boundaries, $\theta \rightarrow \theta_{s-}^{+}$and $\theta \rightarrow \theta_{s+}^{-}$, and the only available solutions 
from the complete set obtained that can possibly adjoin the upstream constant state regions are:

(a) a continuation of the previous constant-state solution,

(b) an irregular weak solution (shear layer).

Exceptional cases where $\rho=0$ or $u_{r}=0$ simultaneously with $u_{\theta}=0$ are excluded. The first case above is the degenerate case of a shear layer with zero strength.

\subsubsection{Solutions downstream of the singularities}

Matching across the shear layer of case $(b)$ of $\S 5.5 .1$, the only remaining possible solution, there are two possibilities in each of the limits taken from the downstream side of the singularities, $\theta \rightarrow \theta_{s-}^{-}$and $\theta \rightarrow \theta_{s+}^{+}$;

(a) $u_{\theta}=0$ with $u_{r}=0$ and we recognize this case as a region of stagnant, potentially non-homenthalpic fluid.

(b) $u_{\theta} \rightarrow 0$ with $u_{r} \neq 0$ and we recognize this as any adjoining constant-state region simultaneously at the condition $u_{\theta} \rightarrow 0$.

An extended region of $u_{\theta}=0$ with $u_{r} \neq 0$ is formally excluded by the solution of $\S 3.2$ that requires $u_{r}=0$ to ensure conservation of mass. Note that in case $(b)$, the adjoining constant-state region is unique, moving away from the singularity since again $\left|\boldsymbol{B}_{z}\right| \neq 0$.

\subsubsection{Simultaneous requirements for both singularities}

Since the arguments of $\S \S 5.5 .1$ and 5.5.2 must apply simultaneously for both singularities, $\theta=\theta_{s \pm}$, and since a solution is physically required for all $\theta$ (see exception below) the only allowable matching conditions are:

(a) that the singular non-homenthalpic regions downstream of $\theta=\theta_{s \pm}$ be contiguous if case $(a)$ of $\S 5.5 .2$ applies,

(b) that if case $(b)$ of $\S 5.5 .2$ applies, then the pre-singularity constant state at $\theta_{s-}^{+}$ be identical to the post-singularity state at $\theta_{s+}^{+}$and vice versa that the pre-singularity constant state at $\theta_{s+}^{-}$be identical to the post-singularity state of $\theta_{s-}^{+}$since each of the constant state regions is unique, with $\left|\boldsymbol{B}_{z}\right| \neq 0$, and since $\left|M_{\theta}\right|$ must increase upstream of each of $\theta=\theta_{s \pm}$. This concomitantly requires that the shear layers adjoining the pre-singularity constant states be one and the same.

These are therefore the only two matching conditions allowed by the conservation equations for the shooting problem of matching the two solutions obtained by integrating in the $\pm \theta$ directions, respectively.

As was the case in $\S 5.3$, a third option (the exception noted above) is that the physical solution required between the downstream singular points $\theta=\theta_{s \pm}$ be determined by a different set of equations, e.g. the equations of elasticity for the behaviour of a solid at the shock intersection point.

\subsubsection{Discussion}

The shear-layer matched solution is well known. Nothing about the steady-state solutions of the conservation equations appears to exclude the stagnant wedge solution to the shooting problem. Although the exclusion of this solution as a formal consequence of plausible assumptions by Glimm et al. (1985) and others is initially justifiable, it is worth noting that same assumptions led Glimm to exclude the solution shown in figure $4(j)$. This solution is familiar and occurs in the free expansion of an inviscid supersonic jet.

A related example of the stagnant wedge solution is the aero-spike phenomenon. A sharp spike inserted into the free-stream flow ahead of a blunt body in supersonic 
flow causes a separation bubble and an oblique shock wave to be formed ahead of the body (Moeckel 1951; Stadler \& Nielsen 1954). The resulting oblique shock waves produce less drag than the strong shock produced in the absence of the spike. Although the tip of the spike represents supersonic flow over an oblique wedge, only a thin spike is required to stabilize the larger-scale stagnant wedge region given appropriate downstream boundary conditions.

In the more restrictive case of conventional shock wave interactions, where the stagnant wedge solution is not observed experimentally, we are forced to eliminate this solution empirically. Despite the current absence of formal entropy, stability or other results that consistently reconcile experimental observations across a range of shock-wave interaction configurations, it remains clear that eventual resolution must come from approaches that go beyond simple elimination of unwanted solutions by assumption.

\subsection{Set of allowable interaction solutions}

Combining the results of $\S \S 5.3-5.5$ we have the allowable solutions in the incident and reflected wave regions, and a rigorous basis for matching the solutions at the downstream singularities. The set of allowable interactions is then given by all permutations of the allowable configurations in each of the regions, with the constraint that the configurations be self-consistent with the properties for each elementary wave established in $\S \S 2-4$. We have noted that figure 4 represents a subset of this complete set of interactions. Full description of the regions of parameter space over which each of the possible permutations may exist requires recourse to bifurcation methods using approaches discussed by Glimm (1988), Keller (1987) and Sanderson \& Sturtevant (1993).

\section{Linearized unsteady formulation}

It is known that shock-wave interaction solutions exhibit bifurcation phenomena (e.g. the point at which 3 shock and 4 shock interaction solutions cross with one of the 4 waves being a degenerate Mach line) and fold point behaviour (e.g. the point of maximum flow deflection angle for a single oblique shock wave where the weak and strong solutions meet). Such bifurcation phenomena are the root cause of the non-existence and non-uniqueness problems discussed in $\S 1$. Results from bifurcation theory (e.g. Keller 1987) formally state that the bifurcation points of steady solutions are associated with sign changes of the eigenvalues of the linearized unsteady problem about the bifurcation point. The value of the current formulation then becomes apparent since we are considering steady-state solutions of the generally unsteady forms (equations (2.5) and (4.3)) in time and one spatial coordinate, $\theta$. It is possible therefore that linearization of the current formulation about the steady-state solutions might lead to results for the open problem of stability for many shock-wave interaction flow fields.

Consider perturbations of the conserved quantities

$$
z(\theta, t)=z^{0}(\theta)+z^{\prime}(\theta, t) .
$$

Expanding the unsteady form (equation (2.5)) about $z^{\prime}=0$, we have

$$
r \boldsymbol{A}_{z}^{0} z_{t}^{\prime}+\left[\boldsymbol{B}_{z}^{0}+\boldsymbol{B}_{z z}^{0} z^{\prime}\right]\left[z_{\theta}^{0}+z_{\theta}^{\prime}\right]+\mathbf{C}^{0}+\mathbf{C}_{z}^{0} z^{\prime}+O\left(z^{\prime 2}\right)=0,
$$


where generally the notation $\boldsymbol{A}_{z}^{0}=\left.\boldsymbol{A}_{z}\right|_{z^{\prime}=0}$ applies. The zeroth-order terms represent the steady-flow solution and to $O\left(z^{\prime}\right)$ we have

$$
r \boldsymbol{A}_{z}^{0} z_{t}^{\prime}+\boldsymbol{B}_{z}^{0} z_{\theta}^{\prime}+\boldsymbol{B}_{z z}^{0} z^{\prime} z_{\theta}^{0}+\mathbf{C}_{z}^{0} z^{\prime} \approx 0 .
$$

Proceeding by the usual method of Laplace transform and Fourier series leads conceptually to a generalized eigenvalue problem for the growth rates for the unsteady modes. Realistically, the working out of the flux derivatives is complicated and consideration must be given to the convergence of the integral transforms involved along with the requirement to use generalized functions for the shock jump discontinuities. An alternative approach would be to use the linearized form in the smooth regions and to match this with boundary conditions provided by a linearized form of the weak solutions (4.3). The stability analyses for one-dimensional shock waves due to Swan \& Fowles (1984) and D'yakov (1954) provide a prototype. Full working out of the details with the necessary rigour lies beyond the scope of the current paper, and we present the suggestion here in the hope that it might be of value to others.

\section{Conclusions}

We have studied the interaction of shock waves in two spatial dimensions in steady flow by considering local solutions of the Euler equations about a point that are constant along each ray and therefore exhibit no length scale. In this way, the conservation equations are reduced to a set of linear ordinary differential equations. Examination of the singularities of this set of equations produces families of solutions that reproduce many standard results in gasdynamics. Weak solutions of the system of equations reproduce classical results for oblique shock waves and formally introduce shear layers as admissible solutions. We show that a requirement for geometric regularity of the intersection of the incident waves and an entropy condition formally restricts the allowable solutions for interacting waves to no more than two incident and two reflected waves. Formal examination of the downstream matching requirements for shock-wave interaction problems shows that shear layer and stagnant wedge matching conditions are the only two possibilities admitted. The steady-state conservation equations do not preclude the existence of the stagnant wedge matching condition. Classical results from gasdynamics are shown to follow in a consistent constructive manner directly from the single assumption that the local solution exhibits no length scale. By retaining the time-dependent terms and linearizing about the steady-flow solutions, an approach is suggested for investigating the open problem of the stability of shock-wave interactions.

The author is grateful for assistance received from the Darryl G. Greenamyer Fellowship and C.L. Powell Fellowship funds. This work was supported by AFOSR Grant Nos. F49620-92-J-0110 and F49620-93-1-0338.

\section{REFERENCES}

Courant, R. \& Friedrichs, K. O. 1948 Supersonic Flow and Shock Waves. Interscience, 1948.

D’yakov, S. P. 1954 On the stability of shock waves. Sov. Phys. J. Exp. Theor. Phys. 27, 288-295.

Glimm, J. 1988 The interaction of non-linear hyperbolic waves. Commun. Pure Appl. Maths 41, $569-590$.

Glimm, J., Klingenberg, K., McBryan, O., Plohr, B., Sharp, D. \& Yaniv, S. 1985 Front tracking and two-dimensional Riemann problems. Adv. Appl. Maths 6, 259-290.

Hornung, H. G. 1986 Regular and Mach reflection of shock waves. Annu. Rev. Fluid Mech. 18, 33. 
Hugoniot, H. 1889 Sur la propagation du mouvement dans les corps et spécialement dans les gaz parfaits. J. l'École Polytech. 58, 1-125.

Keller, H. B. 1987 Numerical Methods in Bifurcation Problems. Springer.

MeYer, T. 1908 Über Zweidimensionale Bewegungsvorgänge in einem Gas, das mit Überschallgeschwindigkeit strömt. Dissertation, Göttingen, Forschungsheft des Vereins deutscher Ingenieure, 62, 31-67.

MoecKel, W. E. 1951 Flow separation ahead of blunt bodies at supersonic speeds. NACA TN 2418, 1951.

RANKINE, W. J. M. 1870 On the thermodynamic theory of waves of finite longitudinal disturbance. Trans. R. Soc. Lond. 160, 277-288.

Roe, P. L. 1981 Approximate Riemann solvers, parameter vectors, and difference schemes. J. Comput. Phys. 43, 357-372.

Samtaney, R. \& Meiron, D. I. 1997 Hypervelocity Richtmyer-Meshkov instability. Phys. Fluids 9, 1783-1803.

Samtaney, R. \& Pullin, D. I. 1996 On initial-value and self-similar soultions of the compressible Euler equations. Phys. Fluids 8, 2650-2655.

SANDERSON, S. R. 1995 Shock wave interaction in hypervelocity flow. Caltech PhD thesis.

Sanderson, S. R., Hornung, H. G. \& Sturtevant, B. 2003 Aspects of planar, oblique and interacting shock waves in an ideal dissociating gas. Phys. Fluids 15, 1638-1649.

Sanderson, S. R. \& Sturtevant, B. 1993 Shock wave interactions in hypervelocity flow. Proc. 19th Intl Symp. on Shock Waves, Marseille, 1993.

Stadler, J. R. \& Nielsen, H. V. 1954 Heat transfer from a hemisphere-cylinder equipped with flow-separation spikes. NACA TN 3287.

Swan, G. W. \& Fowles, G. R. 1984 Shock wave stability. Phys. Fluids 18, 28-35.

TAYlor, G. I. \& Maccoll, J. W. 1933 The air pressure on a cone moving at high speeds - I. Proc. R. Soc. Lond. A 139, 278-297. 